\title{
Combination of synthetic and natural polymers in hydrogel: An impact on drug permeation
}

\author{
Gopa Roy Biswas ${ }^{*}$, Sutapa Biswas Majee, Animikh Roy \\ Division of Pharmaceutics, NSHM College of Pharmaceutical Technology, NSHM Knowledge Campus, Kolkata-Group of Institutions, 124 B.L. Saha Road, \\ Kolkata 700 053, India.
}

\begin{tabular}{l} 
ARTICLE INFO \\
\hline Article history: \\
Received on: $26 / 08 / 2016$ \\
Revised on: 03/10/2016 \\
Accepted on: $23 / 10 / 2016$ \\
Available online: $29 / 11 / 2016$ \\
\hline Key words: \\
Hydrogel, goat abdominal \\
skin, Spreadability, Swelling \\
index, Viscosity, \\
Permeability coefficient.
\end{tabular}

\begin{abstract}
Topical hydrogel preparations are applied on skin to obtain local or systemic action. NSAID's are non-steroidal drugs having excellent anti-inflammatory and analgesic activity but it produces GIT ulceration when used orally. To overcome that problem with oral formulations, many NSAID's are preferred to be administered by topical route. The present investigation is aimed to formulate the hydrogel of Diclofenac potassium with different ratio of Carbopol of different grades along with guar gum for application over the skin. Fourier transform Infrared (FT-IR) spectrophotometer has been used to notice drug - polymer interaction. After getting satisfactory combinations of polymers, hydrogel formulations of Diclofenac potassium were subjected to different physicochemical studies. Evaluation tests for visual appearance, $\mathrm{pH}$, viscosity, spreadability, swelling index etc. were found satisfactory. To investigate the drug permeation kinetics and permeability coefficient from the goat abdominal skin, pieces of goat skin were fixed on the Franz diffusion cell, in a way that the upper surface of abdominal skin faced the donor chamber. The experiment was carried out with $2 \mathrm{gm}$ of the drug loaded hydrogel spreaded on the skin surface at $32{ }^{\circ} \mathrm{C}$ in phosphate buffer pH-5.8. A distinct correlation between $\%$ swelling index and permeability coefficient of the formulations through goat abdominal skin has been observed. With increase in \% swelling index over a period of $8 \mathrm{~h}$ the permeability coefficient decreased. It indicates that swelling of hydrogel forms a sticky, gelatinous mass that retards the permeation of Diclofenac potassium through goat abdominal skin. There are significant co relations between viscosity of hydrogel, $\%$ swelling index and permeation coefficient. It has been found that with increase in viscosity, permeability coefficient increased whereas permeability coefficient of drug decreased with increase in \% swelling index of the formulations.
\end{abstract}

\section{INTRODUCTION}

Hydrogel is a network of polymer chains that are waterinsoluble, sometimes found as a colloidal gel in which water is the dispersion medium. They are superabsorbent (they can contain over $99 \%$ water) natural or synthetic polymers. Hydrogels also possess a degree of flexibility very similar to natural tissue, due to their significant water content. They have played a key role in drug delivery technique. Hydrogels may be defined by different ways. The most common of these is that it is a water-swollen, and cross-linked polymeric network produced

\footnotetext{
* Corresponding Author

E-mail: gopa.biswas@nshm.com
}

by the simple reaction of one or more monomers (Buchholz and Graham, 1998). Another definition is that it is a polymeric material that exhibits the ability to swell and retain a significant fraction of water within its structure, but will not dissolve in water (Enas and Ahmed, 1991).

Hydrogels have received considerable attention in the past 50 years, due to their promises in wide range of applications. The ability of hydrogels to absorb water is due to hydrophilic functional groups attached to the polymeric structure, while their resistance to dissolution arises from cross-links between network chains.

During last two decades, natural hydrogels were gradually replaced by synthetic hydrogels which has got a number of advantages like long service life, high capacity of water absorption, and high gel strength (Yuhui et al., 2013). 
Depending on the properties of the polymer (polymers) used, as well as on the nature and density of the network joints, such structures in an equilibrium can contain various amounts of water; typically in the swollen state, the mass fraction of water in a hydrogel is much higher than the mass fraction of polymer (Veeran and Betageri, 2011).

Hydrogels may be synthesized in a number of chemical ways. These include one-step procedures like polymerization and parallel cross-linking of multifunctional monomers, as well as multiple step procedures involving synthesis of polymer molecules having reactive groups and their subsequent cross-linking, possibly also by reacting polymers with suitable cross-linking agents (Burkert et al., 2007).

Diclofenac potassium is a nonsteroidal anti-inflammatory drug (NSAID) taken or applied for reducing inflammation and it acts as an analgesic that reduces pain in certain conditions (Breen et al., 1986). Diclofenac potassium is available as a generic drug in a number of formulations. Over-the-counter (OTC) use is approved in some countries for minor aches and pains and fever associated with common infections. Inflammatory disorders may include musculoskeletal complaints, especially arthritis, rheumatoid arthritis, polymyositis, dermatomyositis, osteoarthritis, dental pain, TMJ pain, spondylarthritis, ankylosing spondylitis, gout attacks and pain management in cases of kidney stones and gallstones. An additional indication is the treatment of acute migraines (Iwaszkiewicz and Hua., 2014). Diclofenac potassium is used commonly to treat mild to moderate postoperative or posttraumatic pain, in particular when inflammation is also present and is effective against menstrual pain and endometriosis.

The major objectives of the current study was to prepare Diclofenac potassium -loaded hydrogel and to study the effect of ratio of Carbopol of different grades and guar gum used in the formulation on the basic properties (Spreadability, Viscosity, Swelling, etc) of hydrogel and correlations of viscosity and swelling of hydrogel preparations with permeability coefficient of drug . Carbopols have excellent bioadhesive property but if used alone, they may cause irritation in topical application due to their low pH (Ahuja et al., 1997). Its irritant properties can be reduced by combining it with other non-irritant bioadhesive polymers. Hence guar gum has been selected in combination.

\section{MATERIALS AND METHODS}

Carbopol 934p, Carbopol 971p, Carbopol 974p were gift samples of Mylan laboratories Ltd, Hyderabad, India. Guar gum was procured from Qualigens Fine Chemicals, Mumbai, India and API Diclofenac potassium was obtained as gift sample from Windlas Biotech Ltd. Dehradun, Uttaranchal. All the other chemicals were obtained commercially.

\section{Drug-Polymer Interaction study}

Drug-excipient interaction, one of the most essential parameters, is studied before development of the formulations. Diclofenac potassium; polymers and polymer mix with drug was mixed separately with IR grade $\mathrm{KBr}$ in the ratio 1:100 and corresponding pellets were prepared by applying 5.5 metric ton of pressure in a hydraulic press. Polymers were Carbopol (934 p, $971 \mathrm{p}$ and 974 p) and Guar gum. The pellets were scanned over a wave number range of 4000 to $400 \mathrm{~cm}-1$ in FTIR spectroscope (ALPHA T, Bruker, USA).

\section{Preparation of hydrogel:}

Carbopol has very good dispersion ability and forms gels rapidly. Carbopol $934 \mathrm{p}, 971 \mathrm{p}$ and $974 \mathrm{p}$ was dispersed in distilled water alone in $0.2 \% \mathrm{w} / \mathrm{v}$ concentration and in combination with Guar gum at $0.15 \% \mathrm{w} / \mathrm{v}$. Guar gum is used in $25 \% \mathrm{w} / \mathrm{w}$ concentration in the formulation (Table 1).

Carbopol is soluble in water while guar gum produces colloidal dispersion in it. With continuous stirring, $50 \mathrm{mg}$ of drug, Diclofenac potassium was added to it. The mixture was stirred until thickening occurred and then neutralized by drop-wise addition of $50 \%(\mathrm{w} / \mathrm{w})$ Triethanolamine, until a transparent gel appeared. Quantity of Triethanolamine was adjusted to achieve gel with desired $\mathrm{pH}$. Gels were stored for 24 hours at the room temperature to stabilize.

Table 1: Components of hydrogel.

\begin{tabular}{|c|c|c|c|c|c|c|}
\hline Formulation & F1 & F2 & F3 & F11 & F22 & F33 \\
\hline Carbopol 934p (\% w/v) & 0.2 & $\begin{array}{ll}-- \\
-1\end{array}$ & & 0.15 & --- & --- \\
\hline Carbopol 971p (\% w/v) & --- & 0.2 & --- & --- & 0.15 & --- \\
\hline Carbopol 974p (\% w/v) & --- & --- & 0.2 & --- & --- & 0.15 \\
\hline Guar gum (mg) & --- & --- & --- & 50 & 50 & 50 \\
\hline $\begin{array}{l}\text { Drug (Diclofenac } \\
\text { potassium) }(\mathrm{mg})\end{array}$ & 50 & 50 & 50 & 50 & 50 & 50 \\
\hline Triethanolamine $(\mathrm{ml})$ & 0.1 & 0.1 & 0.1 & 0.1 & 0.1 & 0.1 \\
\hline
\end{tabular}

\section{Evaluation of hydrogel \\ Physical appearance}

The physical appearance and homogeneity of the prepared gels were tested by visual observations. The marketed formulation was considered as reference.

\section{Spread ability test}

Spread ability can be determined by applying the gel over an even surface and observed for the gritty nature of the hydrogel if present.

\section{pH determination}

The $\mathrm{pH}$ of the gel formulations was determined by using a $\mathrm{pH}$ meter. For $\mathrm{pH}$ determination, $1 \%$ of hydrogel formulation in deionized water was prepared and $\mathrm{pH}$ was determined at $25^{\circ} \mathrm{C} . \mathrm{pH}$ measurement were done after 1, 7 and 15 days after the hydrogel preparations

\section{Drug content}

For assay of the drug in gels, Diclofenac potassium was extracted from $1 \mathrm{~g}$ of each gel formulations with $20 \mathrm{~mL}$ of phosphate buffer $\mathrm{pH} 5.8$ for $30 \mathrm{~min}$. The content was determined spectrophotometrically at $230 \mathrm{~nm}$. 


\section{Viscosity measurement of hydrogel}

The viscosity of formulated gel bases was determined using Brookfield viscometer (DV-II+Pro) with spindle no. 7 at 100 $\mathrm{rpm}$ at the temperature of $25{ }^{\circ} \mathrm{C}$ (Upendra et al., 2015). The determinations were carried out in triplicate and the average of three reading is recorded.

\section{Swelling Index}

The extent of swelling was measured in terms of $\%$ of weight gained by hydrogel mass. One gm from each formulation was weighed and kept on a sieve placed on a petridish containing $10 \mathrm{ml}$ of $\mathrm{pH} 5.8$ buffer solution. At the end of specified time intervals sieve contains hydrogel were withdrawn from petridish and excess buffer was soaked with tissue paper and weighed. The $\%$ of weight gained by the hydrogel was calculated by using following formula (Needleman and Smale, 1995)

$$
\text { Swelling Index } \left.\%=[(\mathrm{Mt}-\mathrm{Mo}) / \mathrm{Mo})^{* 100}\right]
$$

Where, Mt - weight of formulation at time' $t$ '; Mo - Initial weight of formulation

\section{In-vitro drug diffusion study using dialysis membrane:}

In vitro drug diffusion study was performed through dialysis membrane-50 (Hi Media) by using Franz diffusion cell apparatus with a receptor compartment capacity of $60 \mathrm{ml}$ and cross sectional area of $3.14 \mathrm{~cm}^{2}$. The prepared hydrogel was spreaded over the membrane. It was then mounted in the donor compartment so that the membrane facing towards the receiver compartment. The phosphate buffer $\mathrm{pH} 5.8$ was filled in the receiver compartment. The receptor solution was constantly and continuously stirred using magnetic beads and was maintained at $32 \pm 0.5^{\circ} \mathrm{C}$ by circulating the constant temperature water through outer jacket of the diffusion cells. The samples were withdrawn at different time intervals and analyzed for drug. One $\mathrm{ml}$ of receptor solution was withdrawn and an equal volume of fresh buffer $\mathrm{pH}$ 5.8 was replaced. The samples were analyzed for drug content in spectrophotometer in UV - visible spectrophotometer at $230 \mathrm{~nm}$.

\section{Ex-vivo permeation studies using goat Skin Preparation of goat skin}

Fresh Abdominal skin of goat were collected from slaughterhouse and used in the permeation experiments. Hair from the abdominal region was removed carefully by using hair removing cream; the dermal side of the skin was thoroughly cleaned with distilled water for removing any adhering tissues or blood vessels, it was hydrated in phosphate buffer $\mathrm{pH} 5.8$ for an hour (Posina et al.,2011)

\section{Ex-Vivo permeation study}

It was carried out in Franz diffusion cell as reported by Pullakandam et al. (2009) with slight modification (Pullakandam et al., 2009).

Franz diffusion cell of $60 \mathrm{ml}$ capacity was used for the Ex-vivo skin permeation study. 2 gm of hydrogel was spreaded on the goat abdominal skin facing stratum corneum towards the donor compartment. Then the skin was placed on the receiver compartment of the diffusion cell containing phosphate buffer $\mathrm{pH}$ 5.8. The temperature of the system was maintained at $32^{\circ} \mathrm{C} \pm$ $0.5^{\circ} \mathrm{C}$. The phosphate buffer in the receiver compartment was continuously stirred using the magnetic beads during the experiments. $1 \mathrm{ml}$ of the sample withdrawn at the time interval and it was replaced with $1 \mathrm{ml}$ fresh phosphate buffer. Absorbance of the sample was measured in UV - visible spectrophotometer at $230 \mathrm{~nm}$ against blank.

\section{Statistics}

All the experimental data were assessed by one-way ANOVA followed by Tukey HSD Test using Vassar Stats software (USA). For assessing statistical significance $\mathrm{P}<0.01$ has been considered.

\section{RESULTS AND DISCUSSION}

The major objectives of the current study was to prepare Diclofenac potassium loaded hydrogel and to study the effect of ratio of Carbopol of different grades and guar gum used in the formulation on the basic properties (Spreadability, Viscosity, Swelling, etc) of hydrogel and correlations of viscosity and swelling of hydrogel preparations with permeability coefficient of drug .Diclofenac potassium is a nonsteroidal anti-inflammatory drug (NSAID) taken or applied to reduce inflammation and as an analgesic reducing pain in certain conditions.

After the experiments with different combinations of carbopol and Guar gum and evaluating the different physicochemical parameters, the best polymeric compositions achieved have been reported here. The hydrogel were developed with Carbopol 971p, 914p and 934p alone and in combination with guar gum. Six combinations of hydrogel preparations were taken into considerations.

Drug-excipients interaction is a very important study prior to development of a new formulation. Among the various methodologies available to study the drug-excipient interaction, common approaches are FTIR-spectroscopy, DSC, IR spectra etc (Brittain et al., 1991). FTIR-spectroscopy shows the interaction between the molecules at the level of functional groups (Kotting and Gerwert., 2005). Here drug-excipient interaction was studied using FTIR-spectroscopy. Figure 1A , 1B ,1C show the IR spectra of the Diclofenac potassium, Guar gum, Carbopol 971p, whereas 1D shows the IR spectra of mixture of Diclofenac potassium, Guar gum and Carbopol 971p.

The IR spectra of diclofenac potassium exhib- ited distinctive peaks at 1575.33 and $1577.6 \mathrm{~cm}-1$ owing to $-\mathrm{C}=\mathrm{O}$ stretching of the carboxyl ion and at $744.5 \mathrm{~cm}-1$ because of $\mathrm{C}-\mathrm{Cl}$ stretching. Similar results were obtained by Pignatello et al. (2002). HPMC displayed distinctive peak at $1051 \mathrm{~cm}-1$ due to $\mathrm{C}-$ $\mathrm{OH}$ stretching vibrations, and charac- teristic peaks at $2917.1 \mathrm{~cm}-$ 1 due to $\mathrm{CH}$ aliphatic stretching and $3391.1 \mathrm{~cm}-1$ due to intermolecular hydrogen bonding. Carnauba wax exhibited distinctive peaks at $2914.9 \mathrm{~cm}-1$ due to The IR spectra of 
diclofenac potassium exhib- ited distinctive peaks at 1575.33 and $1577.6 \mathrm{~cm}-1$ owing to $-\mathrm{C}=\mathrm{O}$ stretching of the carboxyl ion and at $744.5 \mathrm{~cm}-1$ because of $\mathrm{C}-\mathrm{Cl}$ stretching. Similar results were obtained by Pignatello et al. (2002). HPMC displayed distinctive peak at $1051 \mathrm{~cm}-1$ due to $\mathrm{C}-\mathrm{OH}$ stretching vibrations, and charac- teristic peaks at $2917.1 \mathrm{~cm}-1$ due to $\mathrm{CH}$ aliphatic stretching and $3391.1 \mathrm{~cm}-1$ due to intermolecular hydrogen bonding. Carnauba wax exhibited distinctive peaks at $2914.9 \mathrm{~cm}-$ 1 due to The IR spectra of Diclofenac potassium peaks observed are at $1572,1271-1089,741 \mathrm{~cm}^{-1}$ owing to $-\mathrm{NH},-\mathrm{C}==\mathrm{O},-\mathrm{Cl}-\mathrm{Cl}$ stretches respectively (Figure 1A). All most similar results were reported by Pignatello et al. (2002). In Figure $1 \mathrm{~B}$ the corresponding peaks are for the $\mathrm{C}=\mathrm{O},-\mathrm{OH}$ stretches. In Carbopol the functional groups present are $-\mathrm{COOH},-\mathrm{OH},-\mathrm{CH}$ and the peaks shown in the IR spectra (Figure 1C) is exclusively for those. In Figure 1D, it has been shown that the peaks of Diclofenac potassium are almost intact in the mixture like $1575 \mathrm{~cm}^{-1}, 1272$ $1090,744 \mathrm{~cm}^{-1}$. In this study no chemical interaction between Diclofenac potassium and polymers were noted.
Some minor changes in the peaks were observed in Figure 1D, which indicates that there might be some physical interaction related to the formation of weak to medium intensity hydrogen bonding between polymers and Diclofenac potassium, but permeation studies showed that this type of interaction has no influence on the release of drug from Carbopol and Guar gum based hydrogel. However mixture of polymers can change the rate of diffusion of drug molecules by changing entanglement in polymeric network. Blend of polymers is known to change the rate of diffusion of drug molecules by varying the entanglement in polymeric network; leading to the change of tortuosity of diffusion pathways (Singh and Fan., 1986). Thus the interaction might be helpful in controlling the release of drug molecules from the hydrogel. From the results it is evident that all gel formulations showed uniform homogeneity and spread ability (Table 2). The physical appearance of the gel formulations was white translucent in nature. The $\mathrm{pH}$ of the developed formulations was in accordance with that of human skin $\mathrm{pH}$ ensuring the acceptability to avoid the risk of irritation upon application.
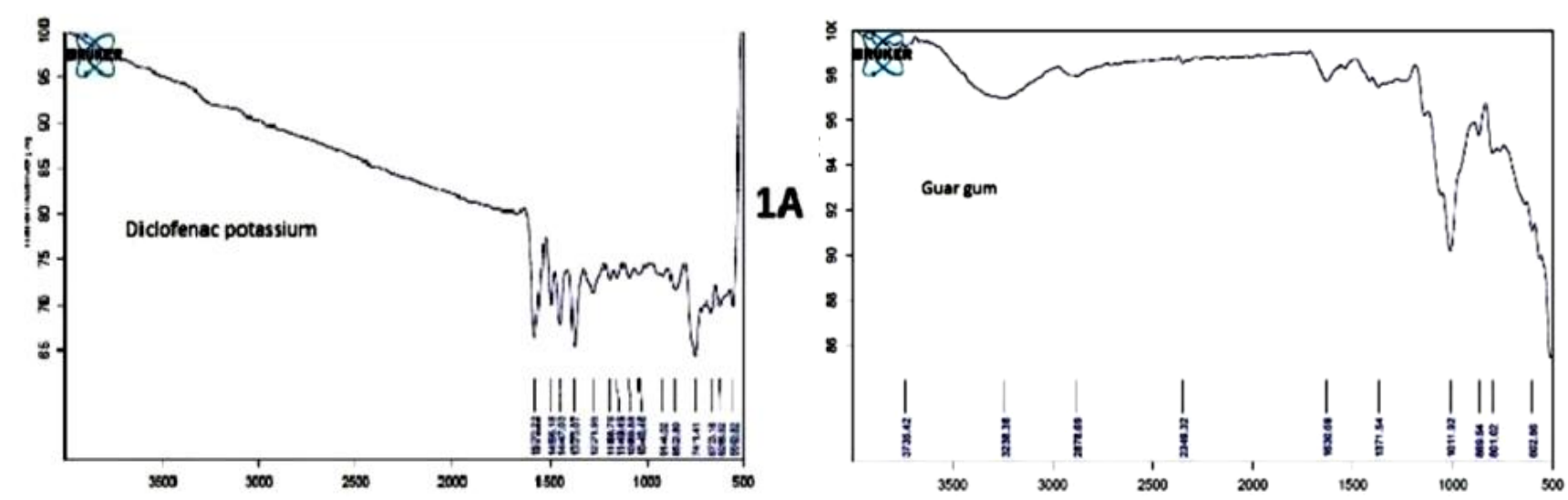

1B
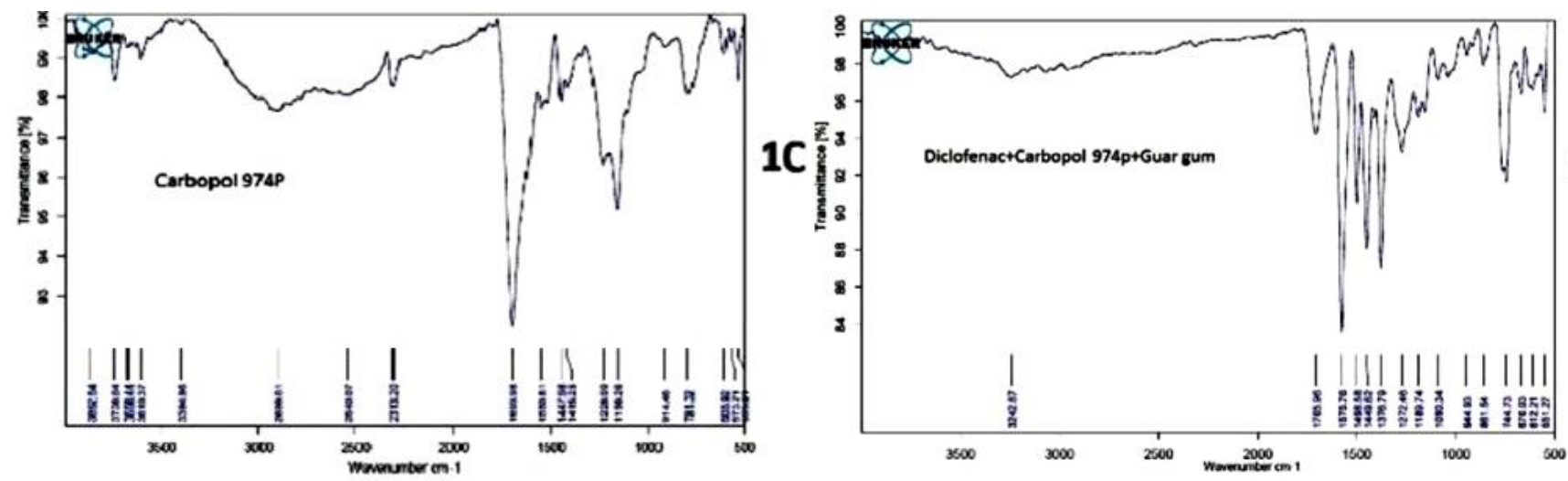

Fig. 1: FTIR spectra of A. Diclofenac potassium B. Guar gum C. Carbopol 974p D. Mixture of Diclofenac potassium, Guar gum, Carbopol 974p. 
Table 2: Physicocmical aspects of the formulation.

\begin{tabular}{|c|c|c|c|c|c|}
\hline Formulation & Visual appearance & Drug content (\%) & Spread ability & $\mathrm{pH}$ & Viscosity (cps) \\
\hline $\mathrm{F} 1$ & Thick, transparent & $99.4 \pm 0.2$ & ++ & $5.78 \pm 0.1$ & 11800 \\
\hline F2 & Thick, transparent & $99.4 \pm 0.2$ & ++ & $5.84 \pm 0.2$ & 15800 \\
\hline F3 & Thick, transparent & $99.4 \pm 0.2$ & ++ & $5.82 \pm 0.2$ & 24200 \\
\hline F11 & Thick, translucent & $99.4 \pm 0.2$ & ++ & $5.83 \pm 0.2$ & 12400 \\
\hline F22 & Thick, translucent & $99.4 \pm 0.2$ & ++ & $5.78 \pm 0.1$ & 21500 \\
\hline F33 & Thick, translucent & $99.4 \pm 0.2$ & ++ & $5.87 \pm 0.2$ & 24500 \\
\hline
\end{tabular}

It is seen that viscosity changes as concentration of polymers changes and among the hydrogel preparations. .It is seen that F33 formulation (Carbopol 974p: Guar gum =3: 1) exhibits higher viscosity (24500 cps) whereas F1 (Carbopol 934p only) exhibits lowest viscosity (11800 cps).

The amount of drug available at the site of application depends on the release from formulation toward the underlying tissues and on the loss (because of diffusion of the drug and erosion of the formulation) toward the external environment (Bonferoni et al., 1999).Some important factors to be considered in the evaluation of the drug permeation across an artificial membrane: the $\mathrm{pH}$ value of the receptor solvent and solubility of the drug in that solvent. The release profile of Diclofenac potassium from hydro gels through dialysis membrane previously demonstrated that this type of membrane does not exert any effect on release (Diez-Sales et al., 2005). The $\mathrm{pH}$ value of the buffer used in release study was chosen to resemble the normal $\mathrm{pH}$ range of skin.

After in-vitro drug diffusion study through dialysis membrane, ex -vivo experiment also conducted to check the corelations between them. Results were quite satisfactory. There were not much deviations.

To investigate the drug permeation kinetics through skin, adult, healthy goat abdominal skin has been selected. If skin permeability is concerned, it is related to body size and physiological life time. According to skin permeability the order is generally mouse $>$ rat > guinea pig > rabbit >Monkey >dog > goat > sheep > pig > human (Walters and Robarts., 1993). Moreover fresh goat skin can be easily collected from slaughter house. Piece of goat abdominal skin was properly processed and fixed on the Franz diffusion cell (Emami et al., 2013). The experiment was thus conducted with 2 gm of drug-loaded hydrogel spreaded over the goat abdominal skin within the donor chamber, $60 \mathrm{ml}$ of phosphate buffer $\mathrm{pH} 5.8$ within the receiver chamber on a magnet stirring device and temperature set at $32^{\circ} \mathrm{C}$. Samples were taken after $30 \mathrm{~m}, 1 \mathrm{~h}, 2 \mathrm{~h}$, $3 \mathrm{~h}$, and $4 \mathrm{~h}$ and up to $6 \mathrm{~h}$, replaced by fresh buffer. The absorbance of the sample was measured spectrophotometrically at $230 \mathrm{~nm}$. The amount of the drug crossed the goat skin could be thus easily calculated.

It has been found that permeation of Diclofenac potassium through goat abdominal skin was minimum in $\mathrm{F} 1$ and maximum in F33 (Figure 2). The drug permeation might be described by zero-order kinetics during the time of study ((ElBadry and Fathy., 2006). The permeability coefficient was calculated using. $\mathrm{P}=\mathrm{K} . \mathrm{Vr} / \mathrm{S}$, where, $\mathrm{S}$ is the effective surface area of abdominal skin of goat skin, $\mathrm{Vr}$ the volume of receiver chamber, $\mathrm{K}$ is the zero order constant, and $\mathrm{P}$ is the permeability coefficient (Biswas et al. , 2015). The permeation profiles for each batch were linear with a mean $\left(\mathrm{R}^{2}\right)$ value of 0.99 . The permeability coefficient found was in a range of $1.11 \times 10^{-3} \mathrm{~cm} / \mathrm{sec}$ to $1.52 \times 10^{-}$ ${ }^{3} \mathrm{~cm} / \mathrm{sec}$.

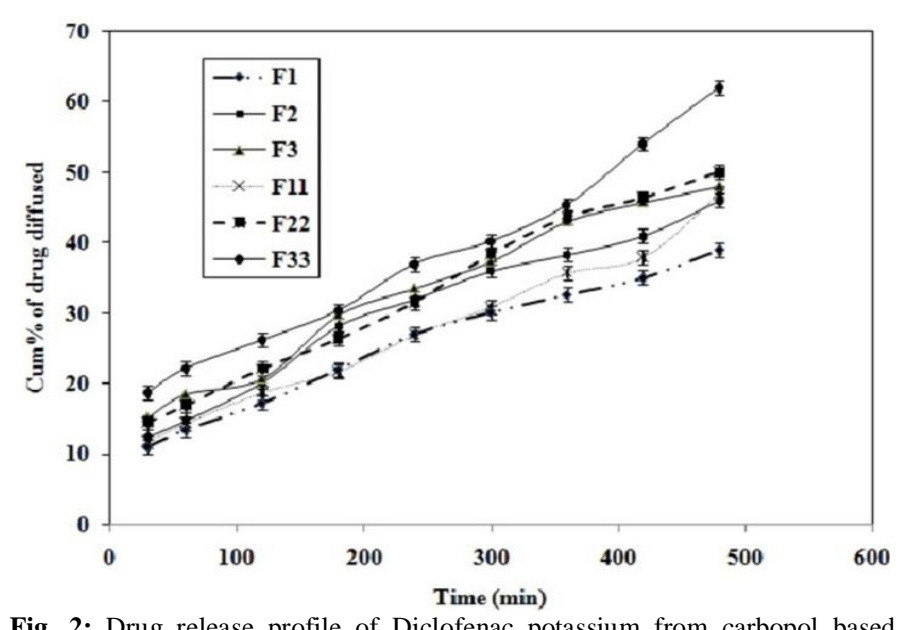

Fig. 2: Drug release profile of Diclofenac potassium from carbopol based hydrogel preparations in phosphate buffer $\mathrm{pH}-5.8,( \pm \mathrm{SD}, \mathrm{N}=6)$

The flux increased considerably in the formulations where guar gum is combined with carbopol than the formulation with carbopol alone. The permeation profiles for each batch was linear with a mean $\left(\mathrm{R}^{2}\right)$ value of 0.9 . F1 (Carbopol 934p: guar gum=1:0) showed flux of $0.748 \pm 0.032 \mathrm{mg} / \mathrm{cm}^{2} / \mathrm{h}$ whereas $\mathrm{F} 11$ (Carbopol934p: guar gum=1:0.25) showed $0.801448 \pm 0.04$ $\mathrm{mg} / \mathrm{cm}^{2} / \mathrm{h}$ of flux value. Highest flux was shown by F33 (Carbopol 974p: guar gum $=1: 0.25$ ) and that is $1.030027 \pm 0.042 \mathrm{mg} / \mathrm{cm}^{2} / \mathrm{h}$.

Among the six best preparations combinations of Carbopol 971p, Carbopol 974p and guar gum offers best drug permeation through abdominal skin of goat skin. Depending on the cumulative $\%$ of drug permeation the formulations can be assigned in the following order F33 $>$ F22 $>$ F3 $>$ F11 $>$ F2 $>$ F1. The result clearly indicates that presence of guar gum initiates drug release hence permeation through skin.

There is a correlation between \% swelling index and permeability coefficient of the formulations through goat abdominal skin (Figure 3). With increase in \% swelling index over a period of $6 \mathrm{~h}$ the permeability coefficient decreased thus the swelling of the formulations is responsible to inhibit the permeation of Diclofenac potassium through skin (Table 3). The hydration rate of the polymer matrix, and thereby the gel formation depends significantly on the ratio of the polymers and thus the viscosity of the polymers (Vazquez et al., 1996) 
.Viscosity plays a significant role in drug permeation (Karakatsani et al., 2010). With increase in viscosity of hydrogel, drug permeation through goat abdominal skin increases. It may be due to increased contact time between skin and drug, which promotes more permeation of drug (Pathan and Setty, 2009).

Table 3: Fluxes and Permeation kinetics of the formulations.

\begin{tabular}{ccc}
\hline Formulation & Flux $\left(\mathbf{m g} / \mathbf{c m}^{2} / \mathbf{h}\right)$ & $\begin{array}{c}\text { Permeability coefficient } \\
\text { Kp }(\mathbf{c m} / \mathbf{s e c})\end{array}$ \\
\hline F1 & $0.748938 \pm 0.032$ & $1.16 \times 10^{-3}$ \\
F2 & $0.890658 \pm 0.025$ & $1.39 \times 10^{-3}$ \\
F3 & $0.946391 \pm 0.029$ & $1.15 \times 10^{-3}$ \\
F11 & $0.801448 \pm 0.021$ & $1.29 \times 10^{-3}$ \\
F22 & $0.965689 \pm 0.04$ & $1.42 \times 10^{-3}$ \\
F33 & $1.030027 \pm 0.042$ & $1.52 \times 10^{-3}$ \\
\hline
\end{tabular}

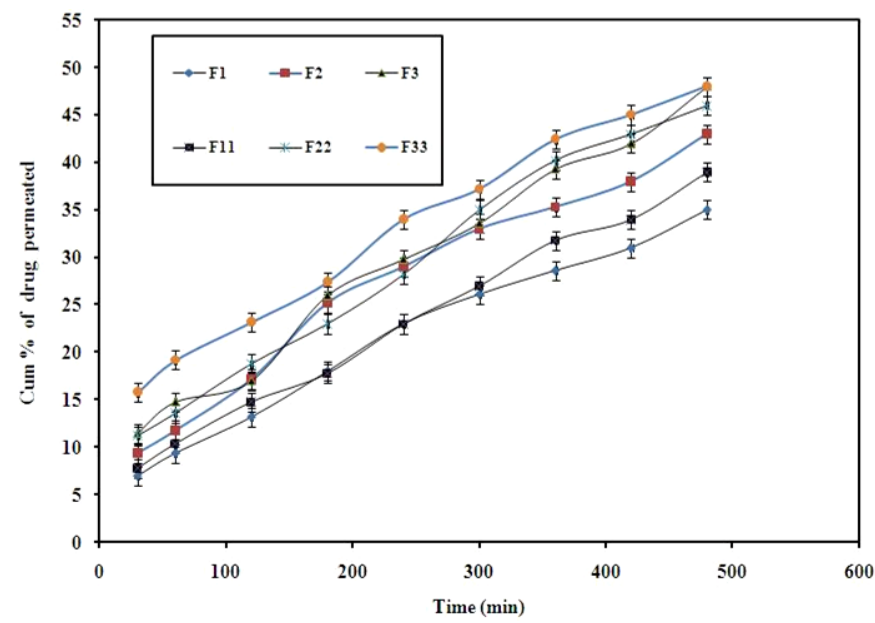

Fig. 3: In-vitro permeation of Diclofenac potassium from carbopol based hydrogel preparations in phosphate buffer $\mathrm{pH}-5.8,( \pm \mathrm{SD}, \mathrm{N}=6)$ )

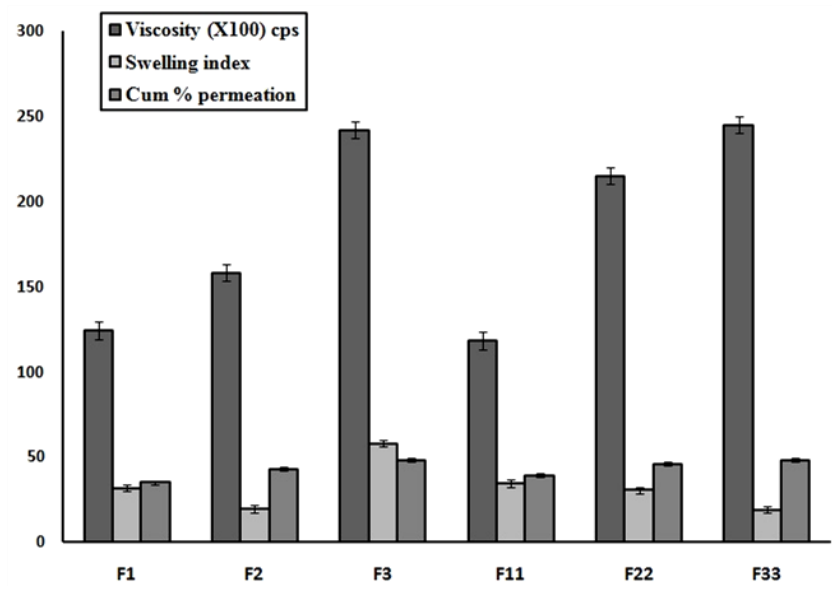

Fig. 4: Comparative study of the hydrogel formulations with respect to their Viscosity, swelling index and permeation. $( \pm \mathrm{SD}, \mathrm{N}=6))$

\section{CONCLUSION}

Diclofenac potassium loaded hydrogel with varying ratio of Carbopol of different grades and guar gum have significant effect on the basic properties (Spreadability, Viscosity, Swelling, etc) of hydrogel. Depending on the cumulative \% of drug permeation the formulations can be assigned in the following order $\mathrm{F} 33>\mathrm{F} 22>\mathrm{F} 3>\mathrm{F} 11>\mathrm{F} 2>\mathrm{F} 1$. The result clearly indicates that presence of guar gum along with Carbopol fastens drug release hence permeation through skin. There are significant correlations between viscosity and swelling of hydrogel preparations with permeability coefficient of drug. With increase in $\%$ swelling index over a period of $6 \mathrm{~h}$ the permeability coefficient decreased thus the swelling of the formulations is responsible to inhibit the permeation of Diclofenac potassium through skin. Viscosity also plays a significant role in drug permeation. With increase in viscosity of hydrogel, drug permeation increases. It may be due to increased contact time between skin and drug which promotes more permeation of drug.

\section{ACKNOWLEDGEMENT}

The authors are thankful to NSHM Knowledge Campus, Kolkata for providing necessary facilities to carry out the research work.

\section{Financial support and sponsorship: Nil.}

Conflict of Interests: There are no conflicts of interest.

\section{REFERENCES}

Ahmed EM., Hydrogel: Preparation, Characterization, and applications: A review. J Adv Res.1991; 17: 297-298.

Ahuja A, Khar RK, and Ali J. Mucoadhesive drug delivery systems. Drug. Dev. Ind. Pharm, 1997; 23: 489-515.

Biswas GR, Chakraborty S, Ghosh N, Majee SB. Fabrication of Bucco-matrix tablets of Amoxicillin trihydrate on the basis of release and permeation kinetics. J Appl Pharm Sci. 2015; 5:.048-052.

Breen EG, McNicholl J, Cosgrove E, McCabe J, Stevens FM . Fatal hepatitis associated with diclofenac. Gut .1986; .27: 1390-3.

Brittain, HG, Bogdanowich SJ , Bugay DE, Vincentis JD, Lewen, G, Newman AW., Physical characterizations of pharmaceutical solids", Pharm Res.1991;.8:. 963-973.

Buchholz FL, Graham AT.1998. Modern superabsorbent polymer technology. New York, USA: Wiley- VCH.

Burkert S, Schmidt T, Gohs U, Dorschner H. t Karl-Friedrich A. Cross-linking of poly ( $\mathrm{N}$-vinyl pyrrolidone) films by electron beam irradiation. Radiat Phys Chem. 2007; 76 : 1324-1328.

Diez-Sales O, Garrigues TM, Herraez JV, Belda R, MartínVillodre A, Herraez M. In vitro percutaneous penetration of penciclovir from solvent systems and Carbopol 971-P hydrogels: Influence of propylene glycol. J Pharm Sci. 2005; 94, 1039-1047.

El-Badry M, Fathy, M. Enhancement of the Dissolution and Permeation Rates of Meloxicam by Formation of Its Freeze-dried Solid Dispersions in Polyvinylpyrrolidone K-30. Drug Dev. Ind. Pharm. 2006; 32: $141-150$.

Emami, J. . ShetabBoushehri M.A, Varshosaz J., and Eisaei A. Preparation and characterization of a sustained release buccoadhesive system for delivery of terbutaline sulfate, Res Pharm Sci.2013;.8.: 219231.

Inayat BP, Setty MC. Chemical penetration enhancers for transdermal drug delivery systems.Trop J Pharm Res. 2009; 8 : 173-179.

Iwaszkiewicz KS, Hua S. Development of an effective topical liposomal formulation for localized analgesia and anti- 
inflammatory actions in the Complete Freund's Adjuvant rodent model of acuteinflammatory pain. Pain Physician. 2014; 17:E719-35.

Kadajji VG, Betageri GV. Water Soluble Polymers for Pharmaceutical Applications. Polymers. 2011; 3: .1972-2009.

Karakatsani M, Dedhiya M, Plakogiannis FM.The effect of permeation enhancers on the viscosity and the release profile of transdermal hydroxypropyl methylcellulose gel formulations containing diltiazem HCl. Drug Dev Ind Pharm.2010; 36:1195-1206.

Kotting C, Gerwert K. Monitoring protein-ligand interactions by time-resolved FTIR difference spectroscopy. Meth Mol Biol. 2005; 305: 261-86.

Needleman IG, Smales FC. In vitro assessment of bioadhesion for periodontal and buccal drug delivery. Biomat. 1995; 617-624.

Pignatello, R., M. Ferro \& G. Puglisi. Preparation of solid dispersions of nonsteroidal anti-inflammatory drugs with acrylic polymers and studies on mechanisms of drug-polymer interactions. AAPS. Pharm. Sci. Tech. 2002; 3: article 10.

Posina A, Sundarapandiyan R, Mohamed T S Saleem .Preparation, in-vitro and in-vivo characterization of transdermal patch containing glibenclamide and atenolol: a combinational approach. Pak. J Pharm. Sci. 2011; 24:155-63.

Pullakandam N, Ashok kJ, Lakshmanaprabu S, Gopal V. Transdermal drug delivery system: an overview, Int J Pharm Sci Rev Res. 2010; 3: 49-55.

Rakde AK, Galgatte UC, Chaudhari PD. In-situ gelling characteristics of Gellan gum at various simulated independent physiological conditions. J Chem pharm Res. 2015; 7: 819-829.
Singh SK, Fan LT. A generalized model for swelling controlled release systems. Biotech Progr.1986; 2:145-56.

Vazquez MJ, Casalderrey M, Gomej-Amoza JG, MartinezPacheco R, Concheiro A. Atenolol release from hydrophilic matrix tablets with Hydroxyl propyl methylcellulose (HPMC) mixtures as gelling agent: effects of the viscosity of HTML mixture. Eur.J.Pharm.Sci.1996; 4: 39-48.

Walters KA., Roberts MS., Veterinary applications of skin in: K.A. Walters, J. Hadgraft (Eds.), Pharmaceutical Skin Penetration Enhancement, Marcel Dekker, New York, 1993, pp. 345-364.

Yuhui Li, Guoyou Huang, Xiaohui Zhang, Baoqiang Li, Yongmei Chen, Tingli Lu,Tian Jian Lu,Feng Xu. Magnetic hydrogels and their potential biomedical applications. Adv Funct Mater. 2013; .23 : 660672.

\section{How to cite this article:}

Biswas GR, Majee SB, Roy A. Combination of synthetic and natural polymers in hydrogel: An impact on drug permeation. J App Pharm Sci, 2016; 6 (11): 158-164. 